$03,05,13$

\title{
Спектроскопия потерь энергии отраженных электронов $\gamma-\mathrm{Fe}_{2} \mathrm{O}_{3}$
}

\author{
(C) А.С. Паршин ${ }^{1}$, Ю.Л. Михлин ${ }^{2}$, Г.А. Александрова ${ }^{1}$ \\ ${ }^{1}$ Сибирский государственный университет науки и технологий им. М.Ф. Решетнева, \\ Красноярск, Россия \\ ${ }^{2}$ Институт химии и химической технологии СО РАН, ФИЦ Красноярский научный центр СО РАН, \\ Красноярск, Россия \\ E-mail: aparshin2010@mail.ru
}

Поступила в Редакцию 23 марта 2021 г.

В окончательной редакции 11 апреля 2021 г.

Принята к публикации 13 апреля 2021 г.

\begin{abstract}
Исследованы спектры потерь энергии отраженных электронов, полученные при энергии первичных электронов в диапазоне 200-3000 eV. Из этих экспериментальных спектров для каждой энергии первичных электронов вычислены спектры сечения неупругого рассеяния электронов как зависимости произведения средней длины неупругого пробега и дифференциального сечения неупругого рассеяния электронов от потерь энергии электронов. Анализ тонкой структуры потерь энергии отраженных электронов проведен разложением спектров сечения неупругого рассеяния электронов в области потерь энергии валентных электронов на элементарные пики. Установлена связь каждого из элементарных пиков с однократными и многократными потерями энергии на возбуждение объемных и поверхностных плазмонов и межзонных переходов электронов из валентной зоны в свободные состояния выше уровня Ферми. Анализ полученных результатов проведен на основе экспериментальных и теоретических литературных данных о зонной структуре $\gamma-\mathrm{Fe}_{2} \mathrm{O}_{3}$.
\end{abstract}

Ключевые слова: электронная спектроскопия, сечение неупругого рассеяния, плазмон.

DOI: $10.21883 /$ FTT.2021.08.51152.053

\section{1. Введение}

Ферримагнитный $\gamma-\mathrm{Fe}_{2} \mathrm{O}_{3}$ (маггемит) находит применение в устройствах магнитной записи информации, датчиках, в качестве катализатора в различных химических процессах [1]. Благодаря своим магнитным свойствам наночастицы маггемита используются в биомедицине для магнитной доставки лекарств [2]. Перспективным направлением является применение этого материала в устройствах спинтроники. При синтезе эпитаксиальных слоев ферритов необходимо учитывать возможность формирования дефектной структуры шпинели на основе $\gamma-\mathrm{Fe}_{2} \mathrm{O}_{3}[3,4]$.

Спектроскопия потерь энергии отраженных электронов позволяет анализировать электронную структуру различных материалов, поскольку в этом методе анализируются потери энергии вторичных электронов, связанные с возбуждением внутренних уровней атомов, межзонными переходами и возбуждением коллективных колебаний пространственного заряда (плазмонов) в твердом теле $[5,6]$. Область с низкими потерями энергии, называемую часто спектроскопией потерь энергии валентных электронов, отражает структуры валентной энергетической зоны и зоны проводимости. Для межзонных переходов валентных электронов максимальные потери энергии электронов определяются разностью энергий между дном валентной зоны и вершиной зоны проводимости, которая для большинства материалов составляет 10-40 eV [7]. Потери энергии на возбуждение объемных и поверхностных плазмонов находятся в пределах $\sim 35 \mathrm{eV}$. Переходы электронов с глубоких уровней в свободные состояния выше уровня Ферми изучаются в спектроскопии потерь энергии глубоких уровней. Потери энергии электронов при возбуждении глубоких уровней составляют десятки и сотни электронвольт и легко могут быть идентифицированы по энергии ионизации соответствующего энергетического уровня атома. В противоположность этому, в спектроскопии потерь энергии валентных электронов в диапазоне малых потерь энергии пики часто обусловлены комбинацией одночастичных межзонных переходов и коллективных плазмонных мод и, как правило, имеют энергетические перекрытия. Это требует применения новых подходов в количественном анализе спектров потерь энергии валентных электронов.

В настоящей работе проведен количественный анализ спектров потерь энергии отраженных электронов $\gamma-\mathrm{Fe}_{2} \mathrm{O}_{3}$ в диапазоне энергий первичных электронов 200-3000 eV. Из экспериментальных спектров потерь энергии вычислены спектры сечения неупругого рассеяния электронов. Для диапазона потерь энергии валентных электронов проведено разложение спектров на элементарные составляющие, как это ранее было сделано для $\mathrm{Fe}[8]$ и других материалов $[9,10]$.

\section{2. Методика эксперимента}

В качестве образца для исследований использован полностью выделенный из породы природный минерал 


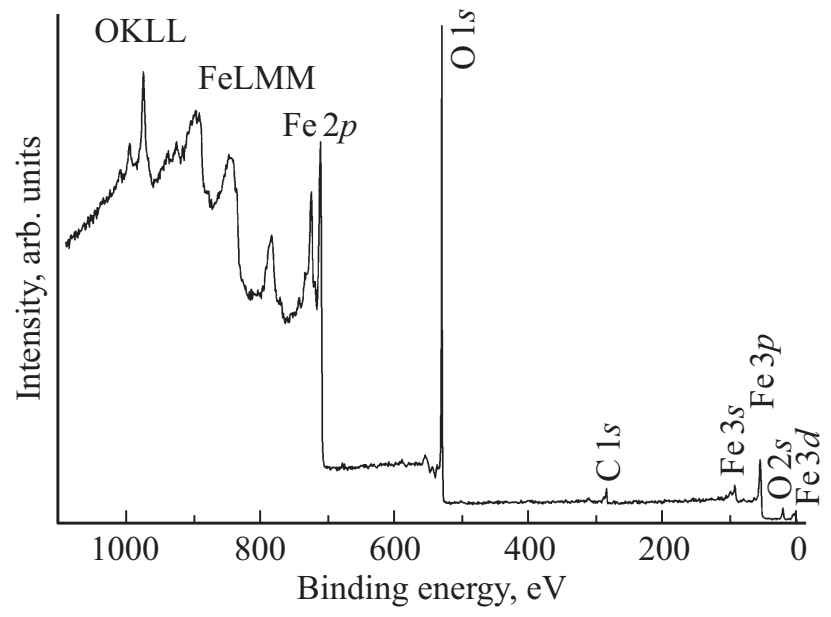

Рис. 1. Обзорный спектр РФЭС $\gamma-\mathrm{Fe}_{2} \mathrm{O}_{3}$.

маггемит. Измерения электронных спектров проводили на сверхвысоковакуумном фотоэлектронном спектрометре SPECS (Германия), при углах падения и детектирования электронов относительно нормали к поверхности образца соответственно 59 и $0^{\circ}$. Спектрометр укомплектован сферическим анализатором энергии электронов, рентгеновской трубкой с двойным анодом как источником рентгеновского излучения, электронной пушкой для возбуждения спектров потерь энергии электронов и растровой ионной пушкой для ионного травления поверхности образца. Удаление поверхностных примесей осуществлялось травлением ионами аргона $\mathrm{Ar}^{+}$(ускоряющее напряжение $2.5 \mathrm{kV}$, ионный ток $15 \mu \mathrm{A}$ ) непосредственно в сверхвысоковакуумной камере спектрометра перед записью электронных спектров. Полнота удаления поверхностных примесей контролировалась по рентгеновским фотоэлектронным спектрам. На рис. 1 приведен обзорный спектр фотоэлектронов образца $\gamma-\mathrm{Fe}_{2} \mathrm{O}_{3}$. Кроме фотоэлектронных и Оже-электронных пиков $\mathrm{Fe}$ и $\mathrm{O}$ присутствует небольшой по интенсивности пик углерода $\mathrm{C} 1 \mathrm{~s}$ слоя углевододородных загрязнений, который практически полностью удалялся ионным травлением до остаточного содержания менее $5 \%$.

\section{3. Экспериментальные результаты}

Экспериментальные спектры потерь энергии отраженных электронов получены при различных энергиях первичных электронов в интервале $200-3000 \mathrm{eV}$ и в интервале потерь энергии $150 \mathrm{eV}$. На рис. 2 приведен спектр при энергии первичных электронов $600 \mathrm{eV}$. Обозначены области, соответствующие потерям энергии на возбуждение валентных электронов и электронов глубоких энергетических уровней атомов.

Пики потерь в области возбуждений глубоких энергетических уровней атомов (пики $M_{1}$ и $M_{2,3}$ на рис. 2) могут быть сравнительно легко идентифицированы, по- скольку на энергии связи внутренних уровней незначительно влияет химическое состояние элементов. Асимметричный пилообразный пик $M_{2,3}$ при энергии потерь приблизительно $57 \mathrm{eV}$ соответствует переходу $3 p$ электронов железа в незанятые состояния выше уровня Ферми. Энергия связи Fe $3 p$ электронов $56.0 \mathrm{eV}$, определенная из спектров РФЭС (рис. 1), согласуется с результатами спектроскопии потерь энергии отраженных электронов. Полученное нами ранее значение энергии потерь пика $M_{2,3}$ чистого железа [8] хорошо согласуется с результатами для $\gamma-\mathrm{Fe}_{2} \mathrm{O}_{3}$. Сравнительно широкий пик $M_{1}$ с потерями энергии приблизительно $93 \mathrm{eV}$ соответствует переходу $3 s$ электронов железа в свободные состояния выше уровня Ферми, что согласуется с результатами измерения из спектров РФЭС энергии связи $\mathrm{Fe} 3 s$ электронов $94.0 \mathrm{eV}$. Спектр потерь энергии валентных электронов может быть использован для определения ширины запрещенной зоны $E_{g}$ [11-13]. Значение $E_{g}$ соответствует началу межзонного электроннодырочного перехода и определяется точкой пересечения аппроксимирующей прямой с максимальным наклоном, близкой к началу спектра, с уровнем фона, как это показано на рис. 3. Определенная таким методом ширина запрещенной зоны $\gamma-\mathrm{Fe}_{2} \mathrm{O}_{3}$ равна $1.9 \mathrm{eV}$ и соответствует литературным данным [14,15].

Значительно сложнее интерпретировать спектры потерь энергии валентных электронов, поскольку в этой области, как указывалось выше, следует ожидать интерференции пиков плазмонных возбуждений и межзонных переходов валентных электронов, наложенных на достаточно большой фон неупругих электронов. Необходимо также учитывать пики кратных потерь энергии. Ранее [8-10], при анализе спектров потерь энергии отраженных электронов ряда металлов и силицидов железа, для вычитания фона неупругих электронов и кратных потерь энергии нами был применен алгоритм вычисления спектров сечения неупругого рассеяния

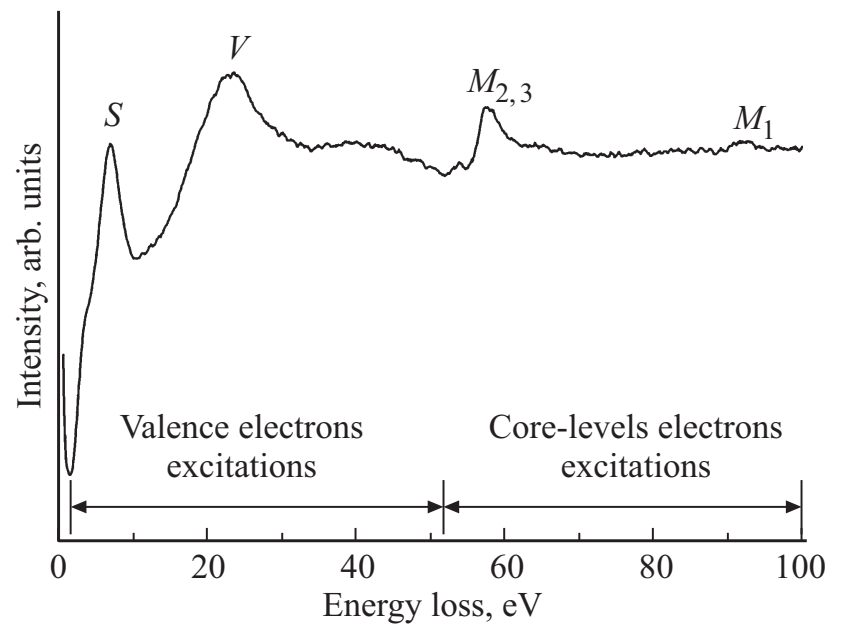

Рис. 2. Спектр потерь энергии отраженных электронов для $\gamma$ - $\mathrm{Fe}_{2} \mathrm{O}_{3}$ при энергии первичных электронов $600 \mathrm{eV}$. 


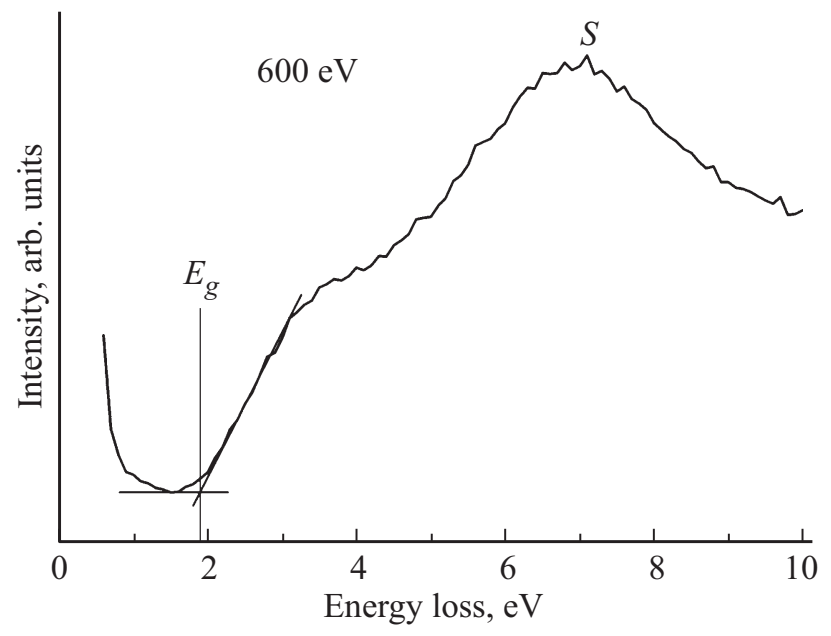

Рис. 3. Определение ширины запрещенной зоны $\gamma-\mathrm{Fe}_{2} \mathrm{O}_{3}$.

электронов ( $K \lambda$-спектров, где $K-$ дифференциальное сечение неупругого рассеяния электронов, $\lambda-$ средняя длина неупругого пробега электронов) из спектров потерь энергии отраженных электронов. Этот алгоритм, предложенный в [16], реализован в программном пакете QUASES ${ }^{\mathrm{TM}}$ XS REELS (Quantitative Analysis of Surfaces by Electron Spectroscopy cross section determined by REELS) [17]. Далее спектры сечения неупругого рассеяния электронов разлагались на элементарные пики, что позволило определить природу отдельных пиков потерь.

В настоящей работе после стандартной процедуры вычисления спектров сечения неупругого рассеяния электронов вычитался остаточный небольшой фон в области потерь энергии $0-55 \mathrm{eV}$ по методу Ширли [18], поскольку, как отмечалось в работах $[16,19]$, несоответствие модели транспорта электронов в твердом теле и реальных условий эксперимента приводит к нефизическому отрицательному значению сечения рассеяния при энергиях потерь, соответствующих сумме потерь энергий на возбуждения объемного и поверхностного плазмона для $\mathrm{Al}$ и $\mathrm{Si}$, а также присутствию в спектрах сечения неупругого рассеяния электронов в $\mathrm{Al}$ пика двукратного возбуждения объемного плазмона.

Спектры сечения неупругого рассеяния электронов для пяти значений энергий первичных электронов после вычитания остаточного фона в области потерь энергии валентных электронов приведены на рис. 4. С изменением энергии первичных электронов интенсивности и энергетические положения двух максимумов, обозначенных на рис. 2 и 4 , как $S$ и $V$, ведут себя поразному. Интенсивность максимума $S$ монотонно уменьшается с возрастанием энергии первичных электронов, а энергия потерь, соответствующая этому максимуму, практически остается постоянной. Интенсивность максимума $V$ стремится при увеличении энергии первичных электронов к постоянной величине, а положение этого максимума заметно смещается в сторону высоких зна- чений энергий потерь. Такое поведение максимума $V$ можно качественно объяснить перераспределением интенсивностей неразрешенных по энергии объемного и поверхностного пиков, составляющих результирующий максимум $B$, с изменением энергии первичных электронов, как это было показано в работе [8]. Основной вклад в максимум $S$ вносят потери поверхностной природы.

Количественный анализ спектров сечения неупругого рассеяния валентных электронов проведен разложением исходного спектра на элементарные лоренцовоподобные пики, введенные Тоугаардом для описания универсальных классов сечения неупругого рассеяния электронов [20]. Спектр сечения неупругого рассеяния электронов аппроксимировали суммой трехпараметрических функций Тоугаарда

$$
\lambda K(T)=\sum_{i}^{n}\left(B T /\left(\left(C-T^{2}\right)^{2}+D T^{2}\right)\right) .
$$

Здесь: $T-$ потери энергии, а $B, C$ и $D-$ подгоночные параметры, которые подбираются из условия наилучшего согласия теоретических расчетов с экспериментом. Средняя длина свободного пробега электронов существенно зависит от их энергии [21], что позволяет из зависимостей спектров сечения неупругого рассеяния электронов от энергии первичных электронов проанализировать вклады поверхностных и объемных возбуждений в потери энергии электронов. Дифференциальное сечение неупругого рассеяния электронов сильно зависит от потерь энергии $T$, но незначительно от $E$ [22], поэтому в первом приближении можно пренебречь зависимостью $K$ от $E$. Количество пиков в разложении $n=9$. Увеличение числа пиков сверх этого значения не приводит к существенному изменению энергетических положений и амплитуд оставшихся пиков или приводит к недостоверным значениям подгоночных параметров. На рис. 5 приведен пример разложения спектра сечения

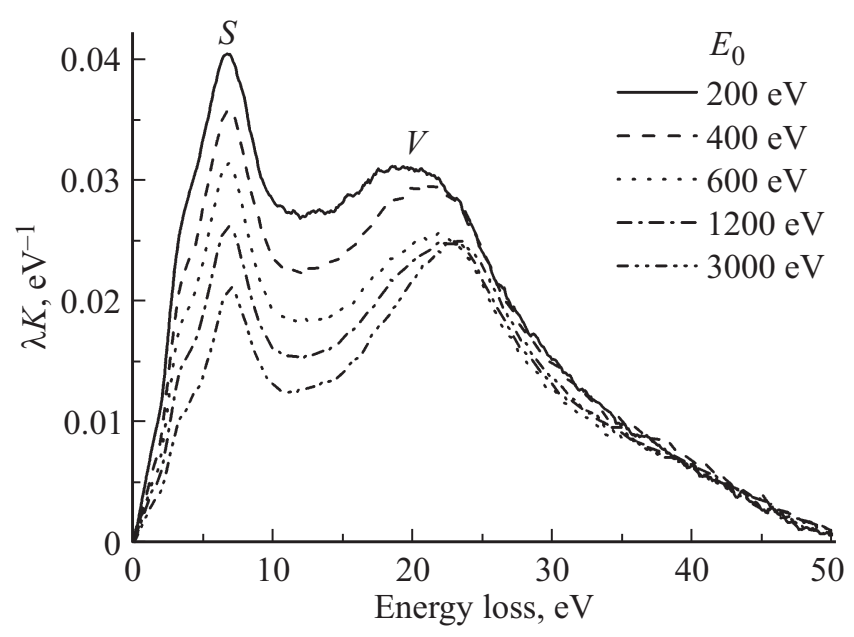

Рис. 4. Спектры сечения неупругого рассеяния электронов для разных энергий первичных электронов. 


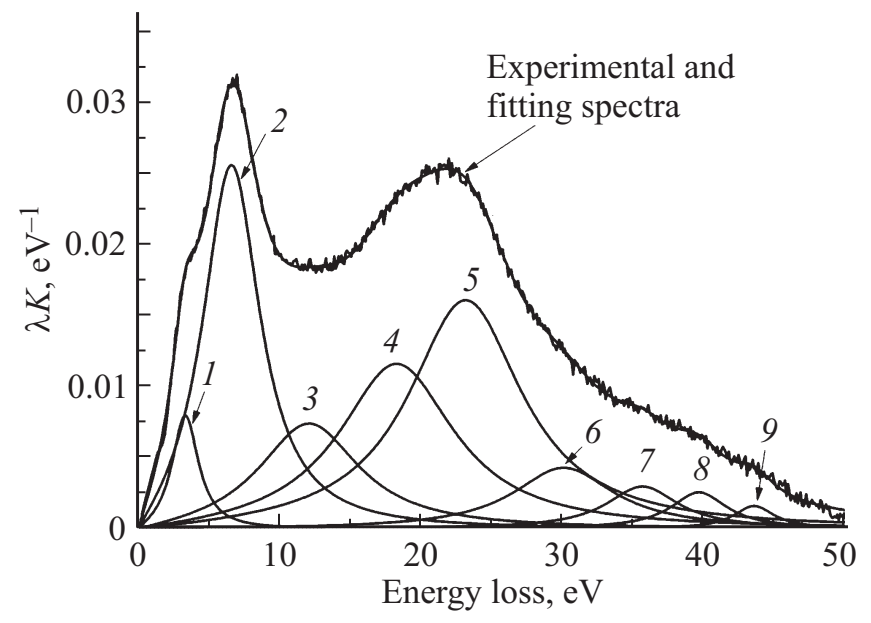

Рис. 5. Разложение спектра сечения неупругого рассеяния электронов $\gamma-\mathrm{Fe}_{2} \mathrm{O}_{3}$ на элементарные пики при энергии первичных электронов $600 \mathrm{eV}$.

неупругого рассеяния электронов для энергии первичных электронов $600 \mathrm{eV}$.

Зависимости интенсивностей и энергетических положений трех пиков разложения, дающих основной вклад в спектр сечения неупругого рассеяния электронов, от энергии первичных электронов приведены на рис. 6 . С увеличением энергии первичных электронов от 200 до $3000 \mathrm{eV}$ средняя длина свободного пробега электронов в $\mathrm{Fe}_{2} \mathrm{O}_{3}$ возрастает от 0.7 до $4.7 \mathrm{~nm}$ [23]. Значительное уменьшение амплитуды низкоэнергетического пика 2 (здесь и далее нумерация пиков соответствует рис. 5) с увеличением энергии первичных электронов свидетельствует о его поверхностной природе. В противоположность этому, амплитуда высокоэнергетического пика 5 в пределах погрешности измерений не меняется с увеличением глубины анализа, что характерно для объемных потерь.

Значения энергий всех подгоночных пиков для различных энергий первичных электронов, их средние величины и среднеквадратичные отклонения приведены в таблице. Относительные среднеквадратичные отклонения энергий подгоночных пиков колеблются от $1.3 \%$ для пика 5 до $3.1 \%$ для пика 7.

Энергия потерь основного пика 5 в разложении спектров сечения неупругого рассеяния электронов остается постоянной с изменением энергии первичных электронов и, следовательно, глубины анализа и близка к энергии объемного плазмона металлического железа [8]. Интенсивность этого пика, как следует из рис. 6, $c$, практически не меняется с энергией первичных электронов. Детальный количественный анализ зависимости амплитуды пика от энергии первичных электронов показал, что среднеквадратичное отклонение этой величины от среднего значения не превышает 7\%. Следовательно, потери энергии $23.4 \mathrm{eV}$ можно связать с возбуждением в $\gamma-\mathrm{Fe}_{2} \mathrm{O}_{3}$ объемного плазмона. В соответствии с класси- ческим соотношением между энергиями объемного $E_{P V}$ и поверхностного $E_{P S}$ плазмонов $E_{P V} / E_{P S}=\sqrt{2}$ в спектре должен быть пик с энергией потерь приблизительно $16.5 \mathrm{eV}$. Однако в разложении пика с такой энергией потерь нет. Возможно, элементарный пик 4 с энергией потерь $18.6 \mathrm{eV}$ представляет собой наложение пиков поверхностного плазмона и пика потерь одноэлектрон-
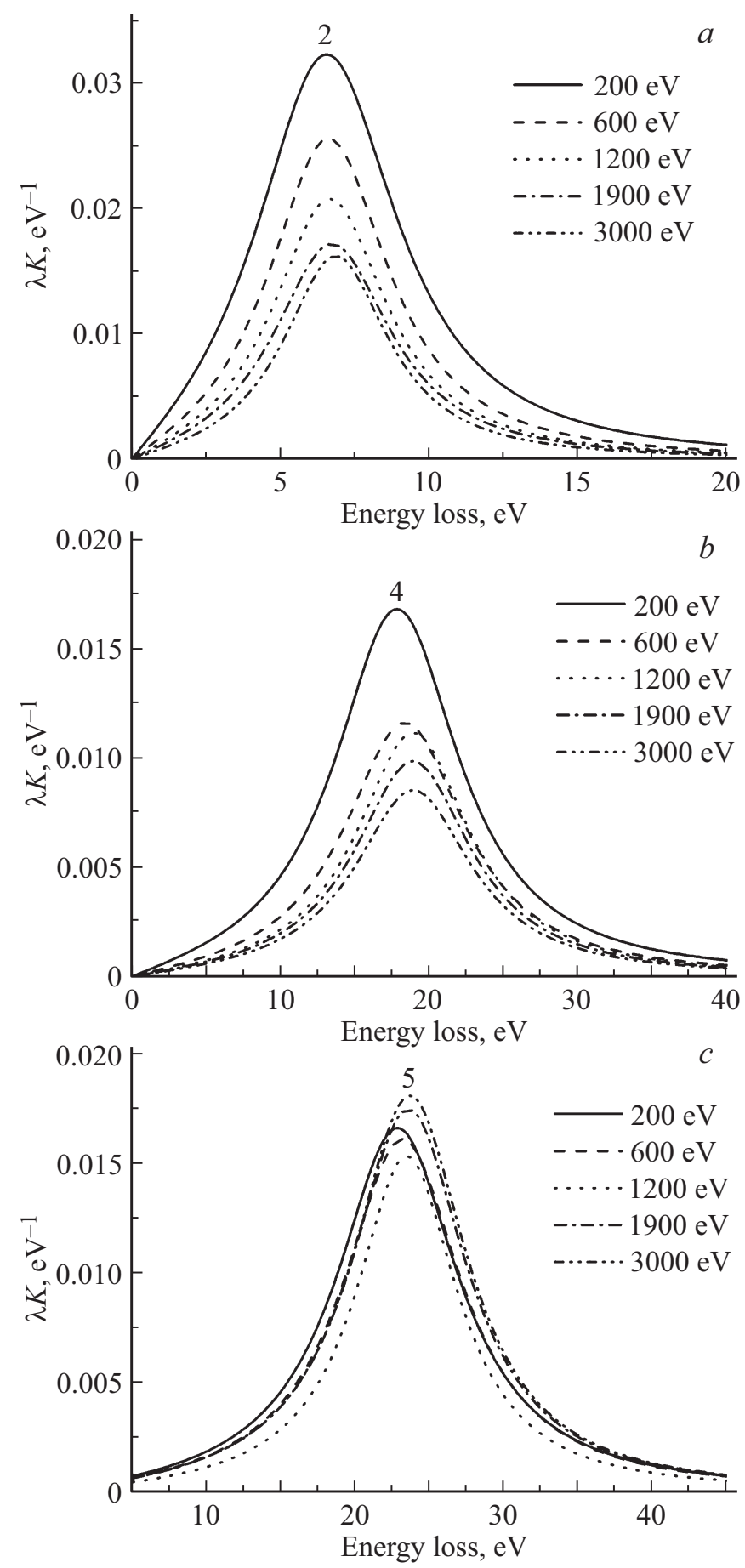

Рис. 6. Зависимости второго $(a)$, четвертого $(b)$ и пятого $(c)$ элементарных пиков разложения от энергии первичных электронов. 
Энергии потерь подгоночных пиков (в еV)

\begin{tabular}{c|c|c|c|c|c|c|c|c|c}
\hline$E_{0}, \mathrm{eV}$ & 1 & 2 & 3 & 4 & 5 & 6 & 7 & 8 & 9 \\
\hline 200 & 3.40 & 6.55 & 12.19 & 17.79 & 22.85 & 28.88 & 33.25 & 37.68 & 42.30 \\
\hline 300 & 3.35 & 6.56 & 12.44 & 18.19 & 23.05 & 30.42 & 35.58 & 40.35 & 44.12 \\
\hline 400 & 3.35 & 6.60 & 12.55 & 18.49 & 23.22 & 30.70 & 37.19 & 41.49 & 44.88 \\
\hline 600 & 3.34 & 6.56 & 12.12 & 18.31 & 23.19 & 30.13 & 35.70 & 39.70 & 43.60 \\
\hline 900 & 3.44 & 6.70 & 12.40 & 18.82 & 23.45 & 30.16 & 35.86 & 39.73 & 43.35 \\
\hline 1200 & 3.37 & 6.65 & 12.41 & 18.87 & 23.46 & 28.87 & 33.96 & 39.24 & 43.66 \\
\hline 1500 & 3.39 & 6.64 & 12.37 & 18.80 & 23.58 & 30.04 & 35.46 & 39.77 & 43.98 \\
\hline 1900 & 3.35 & 6.72 & 12.64 & 18.85 & 23.59 & 30.29 & 36.10 & 40.47 & 44.57 \\
\hline 2500 & 3.39 & 6.80 & 12.20 & 19.15 & 23.76 & 29.85 & 35.16 & 39.63 & 43.80 \\
\hline 3000 & 3.48 & 6.86 & 12.17 & 18.88 & 23.68 & 30.15 & 35.20 & 39.33 & 42.82 \\
\hline Среднее & 3.4 & 6.7 & 12.4 & 18.6 & 23.4 & 30.0 & 35.4 & 39.7 & 43.7 \\
\hline Отклонение & 0.05 & 0.1 & 0.2 & 0.4 & 0.3 & 0.6 & 1.1 & 1.0 & 0.8
\end{tabular}

ного межзонного перехода. Косвенным подтверждением в пользу этого предположения является тот факт, что двукратные потери энергии $39.9 \mathrm{eV}$ на возбуждение объемного плазмона с энергией $23.4 \mathrm{eV}$ и поверхностного плазмона с энергией $16.5 \mathrm{eV}$ близки к энергетическим потерям $39.7 \mathrm{eV}$, характеризующими пик 8 (таблица). В пользу этого предположения свидетельствует и то, что амплитуда пика 4 с увеличением энергии первичных электронов от 200 до $3000 \mathrm{eV}$ монотонно уменьшается приблизительно в 2 раза (рис. $6, b$ ), что характерно для пиков поверхностных потерь.

Потери энергии на возбуждение межзонных переходов определяются структурой плотности электронных состояний валентной зоны и незаполненных состояний выше уровня Ферми. Результаты экспериментальных исследований структуры валентной зоны $\gamma$ - $\mathrm{Fe}_{2} \mathrm{O}_{3}$ методом рентгеновской фотоэлектронной спектроскопии приведены в работах [24-29]. Во всех исследованиях отмечается, что фотоэлектронный спектр валентной зоны состоит из широкой полосы в интервале приблизительно $0-10 \mathrm{eV}$. В [24,25] показано, что основная полоса спектра валентной зоны имеет трехпиковую структуру с максимумами приблизительно 3,5 и $7 \mathrm{eV}$. Кроме основной полосы в [25] наблюдали сателлитную полосу в области $10-17 \mathrm{eV}$ фотоэлектронного спектра $\gamma-\mathrm{Fe}_{2} \mathrm{O}_{3}$. Сателлитные структуры в фотоэлектронных спектрах обусловлены гибридизацией $\mathrm{Fe} 3 d-\mathrm{O} 2 p$ орбиталей. Расчетные спектры валентной зоны в рамках кластерной модели [25] хорошо воспроизводят трехпиковую структуру валентной зоны и пик сателлитной полосы, наблюдаемый экспериментально при энергии приблизительно $17.1 \mathrm{eV}$. Теоретические расчеты электронной структуры $\gamma-\mathrm{Fe}_{2} \mathrm{O}_{3}$, основанные на теории функционала плотности $[15,30]$, показывают, что вершина валентной

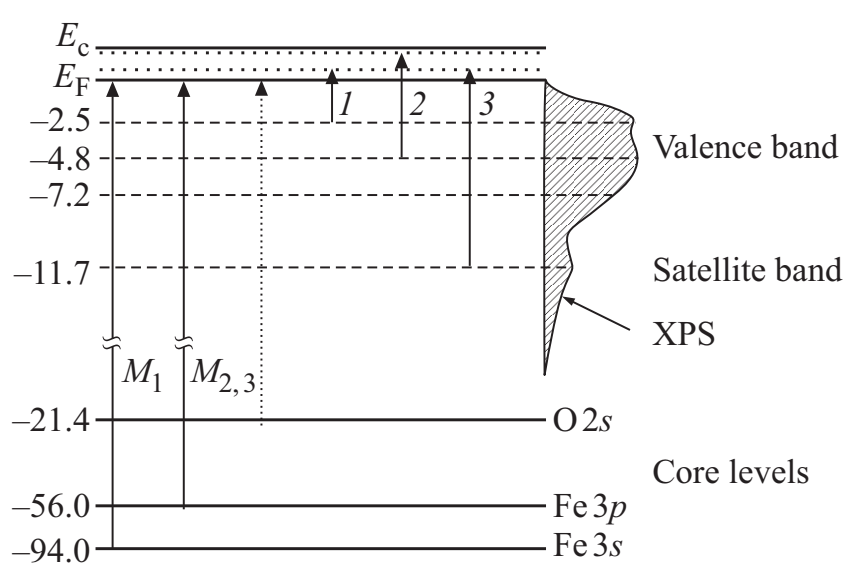

Рис. 7. Схема возбуждения электронов внутренних уровней и валентной зоны.

зоны в основном имеет характер О $2 p$, а занятые $3 d$ уровни $\mathrm{Fe}$ лежат примерно на $6-9 \mathrm{eV}$ ниже уровня Ферми. Уровень Ферми для объемного маггемита расположен вблизи потолка валентной зоны. Эти результаты согласуются с результатами рассмотренных выше экспериментальных исследований фотоэлектронных спектров валентной зоны $\gamma-\mathrm{Fe}_{2} \mathrm{O}_{3}$.

На рис. 7 представлена упрощенная схема, иллюстрирующая межзонные переходы и возбуждения электронов внутренних уровней в $\gamma-\mathrm{Fe}_{2} \mathrm{O}_{3}$. Кривая справа представляет схематически структуру плотности состояний электронов валентной зоны в соответствии с фотоэлектронными спектрами (XPS) [25]. Штриховые линии соответствуют максимумам трехпиковой структуры спектра валентной зоны и сателлитного пика согласно результатам [23]. Сплошными прямыми на диаграмме 
обозначены энергии связи внутренних уровней железа и кислорода. $E_{\mathrm{F}}$ и $E_{c}-$ энергии Ферми и дна зоны проводимости соответственно. Вертикальные линии иллюстрируют межзонные переходы, объясняющие природу пиков потерь энергии электронов, которые регистрируются в спектрах потерь энергии электронов $\gamma-\mathrm{Fe}_{2} \mathrm{O}_{3}$. Спектры потерь энергии электронов, обозначенные на рис. 2 как $M_{1}$ и $M_{2,3}$, обусловлены потерями на возбуждения внутренних уровней $\mathrm{Fe} 3 s$ и $\mathrm{Fe} 3 p$ соответственно. Пики 1,2 и 3 (рис. 5 и таблица), демонстрирующие поверхностную природу, можно связать с межзонными переходами электронов из валентной зоны в свободные поверхностные состояния, расположенные в запрещенной зоне с максимумами плотности 0.7 и $1.8 \mathrm{eV}$ выше уровня Ферми. Эти максимумы обозначены на рис. 7 пунктирными прямыми. Возможны потери энергии, связанные с возбуждением уровня кислорода $2 s$ с энергией связи $21.4 \mathrm{eV}$ [25] (штриховая вертикальная линия на рис. 7), результатом которых будет дополнительный пик потерь, который, как указывалось выше, в сумме с пиком потерь на возбуждение поверхностного плазмона приводит к регистрируемому в спектре пику 4 с энергией потерь $18.6 \mathrm{eV}$. Элементарные пики разложения, с энергиями потерь 30-45eV, также можно связать с кратными потерями (рис. 5 и таблица). Суммарные потери на возбуждение объемного плазмона и межзонного перехода 2 (рис. 7) $23.4+6.7=30.1 \mathrm{eV}$ близки к потерям пика $630.0 \mathrm{eV}$ (см. таблицу.). Аналогично можно показать, что пик 7 соответствует кратным потерям на возбуждения объемного плазмона и межзонного перехода 3. Пик 8 , как указывалось выше, связан с кратными потерями на возбуждение объемного и поверхностного плазмонов. Потери с энергией $43.7 \mathrm{eV}$ (пик 9) можно интерпретировать как кратные потери возбуждения объемного плазмона и одночастичного возбуждения уровня О $2 s$.

\section{4. Заключение}

Экспериментальные спектры потерь энергии отраженных электронов $\gamma$ - $\mathrm{Fe}_{2} \mathrm{O}_{3}$ для энергий первичных электронов в диапазоне $200-3000 \mathrm{eV}$ интерпретированы на основе механизмов потерь на коллективные возбуждения электронов валентной зоны (плазмонов) и одноэлектронные переходы с внутренних уровней и валентной зоны в свободные состояния выше уровня Ферми. Тонкая структура потерь энергии валентных электронов изучена разложением спектров сечения неупругого рассеяния электронов этой области на элементарные пики. Определен механизм потерь каждого пика разложения. Установлено существование свободных поверхностных состояний электронов в запрещенной зоне с максимумами плотности приблизительно на 0.7 и $1.8 \mathrm{eV}$ выше уровня Ферми. На основе полученных экспериментальных результатов предложена упрощенная схема, иллюстри- рующая межзонные переходы валентных электронов и возбуждения электронов внутренних уровней в $\gamma-\mathrm{Fe}_{2} \mathrm{O}_{3}$.

\section{Финансирование работы}

Работа выполнена с использованием оборудования Красноярского регионального центра коллективного пользования ФИЦ КНЦ СО АН.

\section{Конфликт интересов}

Авторы заявляют, что у них нет конфликта интересов.

\section{Список литературы}

[1] R. Dronskowski. Adv. Func. Mater. 11, 1, 27 (2001)

[2] Q.A. Pankhurst, J. Connolly, S.K. Jones, J. Dobson. J. Phys. D 36, 13, R167 (2003).

[3] А.С. Паршин, Н.С. Чистяков. Изв. АН СССР. Неорган. материалы. 17, 2, 346 (1981).

[4] A.S. Parshin, B.E. Blekher, K.P. Polyakova. Int. J. Mod. Phys. B 7, 550 (1993).

[5] H. Raether. Excitation of Plasmons and Interband Transitions by Electrons, Springer (1980). 195 p.

[6] R.F. Egerton. Electron Energy Loss Spectroscopy in the Electron Microscopy, 3nd ed. Springer (2011). 491 p.

[7] C.R. Brundle, C.A. Evans, S. Wilson. Enciclopedia of Materials Characterization: Surfaces, Interfaces, Thin Films. Buttrworth-Heinemann (1992). P. 326.

[8] А.С. Паршин, А.Ю. Игуменов, Ю.Л. Михлин, О.П. Пчеляков, В.С. Жигалов. ФТТ 58, 5, 881 (2016).

[9] А.С. Паршин, А.Ю Игуменов, Ю.Л. Михлин, О.П. Пчеляков, А.И. Никифоров, В.А. Тимофеев. ФТП 49, 4, 435 (2015).

[10] A.S. Parshin, A.Yu. Igumenov, Tu.L. Mikhlin, O.P. Pchelyakov, V.S. Zhigalov. IOP Conf. Ser.: Mater. Sci. Eng. 122, 1, 012025(7) (2016).

[11] H. Jin, S.K. Oh, H.J. Kang, S.W. LeeY, S. Lee, M.-H. Cho. Appl. Phys. Lett. 87, 21, 212902 (2005).

[12] M.L. Huang, Y.C. Chang, Y.H. Chang, T.D. Lin, J. Kwo, M. Hong. Appl. Phys. Lett., 94, 5, 052106 (2009).

[13] D. Tahir, E.K. Lee, S.K. Oh, T.T. Tham, H.J. Kang, H. Jin, S. Heo, J.C. Park, J.G. Chung, J.C. Lee. Appl. Phys. Lett. 94, 21, 212902 (2009).

[14] M.I. Litter, M.A. Blesa. J. Chem. 70, 9, 2502 (1992).

[15] R. Grau-Crespo, A.Y. Al-Baitai, I. Saadoune, N.H. De Leeuw. J. Phys.: Condens. Matter 22, 25, 255401 (2010).

[16] S. Tougaard, I. Chorkendorff. Phys. Rev. B, 35, 13, 6570 (1987).

[17] S. Tougaard. http:// www.quases.com.

[18] Practical Surface Analysis: By Auger and $X$-ray Photoelectron Spectroscopy / Eds. D. Briggs, M.P. Seah. Wiley, Chichester (1983). $533 \mathrm{p}$.

[19] S. Tougaard, J. Kraaer. Phys. Rev. B 43, 2, 1651 (1991).

[20] S. Tougaard. Surf. Interf Anal. 25, 3, 137 (1997).

[21] L.C. Feldman, J.W. Mayer. Fundamentals of Surface and Thin Film Analysis, Elsevier Science, N.Y. (1986). 352 p. 
[22] S. Tougaard, P. Sigmund. Phys. Rev. B 25, 7, 4452 (1992).

[23] S. Tanuma, C.J. Powell, D.R. Penn. Surf. Interf. Anal. 17, 13, 911 (1991).

[24] N.S. McIntyre, D.G. Zetaruk. Anal. Chem., 49, 11 (1977).

[25] T. Fujii, F.M.F. de Groot, G.A. Sawatzky, F.C. Voogt, T. Hibma, K. Okada. Phys. Rev. B 59, 4, 3195 (1999).

[26] L. Minati, V. Micheli, B. Rossi, C. Migliaresi, L. Dalbosco, G. Bao, S. Hou, G. Speranza. Appl. Surf. Sci. 257, 24, 10863 (2011).

[27] D. Flak, A. Braun, B.S. Mun M. Döbeli T. Graule, M. Rekas. Proc. Eng. 47, 257 (2012).

[28] T. Radu, C. Iacovita, D. Benea, R. Turcu. Appl. Surf. Sci. 405, 337 (2017).

[29] D.N.G. Krishna, C. Anushree, R.P. George. John Philip. Appl. Surf. Sci. 462, 932 (2018).

[30] Y.L. Bentarcurt M. Calatayud, J. Klapp, F. Ruette. Surf. Sci. 677, 239 (2018).

Редактор Т.Н. Василевская 